\title{
NUMERICAL ANALYSIS OF THREE- DIMENSIONAL NATURAL CONVECTION IN A CLOSED RECTANGULAR CAVITY UNDER CONDITIONS OF RADIANT HEATING AND CONJUGATE HEAT EXCHANGE
}

\author{
Alexander E. Nee ${ }^{1, *}$ \\ ${ }^{1}$ National Research Tomsk Polytechnic University, 634050, Tomsk, Russia
}

\begin{abstract}
The numerical simulation results of three-dimensional natural convection in a closed cavity were presented under conditions of the bottom horizontal solid-fluid interface radiant heating and conjugate heat exchange. Conservation equations of mass, momentum, and energy were formulated in terms of vorticity vector - vector potential - temperature dimensionless variables and solved by means of the finite difference method. It was found that the heat transfer process under study had a significant unsteady nature. According to the results of conjugate heat exchange integral analysis, it was shown that similar trends of mean Nusselt numbers versus dimensionless time were formed for both two and three dimensional problem formulations.
\end{abstract}

\section{Introduction}

Investigation of convective heat transfer in closed cavities plays an important role for science and engineering. Research of heat transfer regularities in technical systems and technological processes contributes to increasing of their energy performance. However, to conduct full-scale experiment in order to reveal the basic heat exchange characteristics is not always possible to perform under operating conditions of thermal engineering equipment. In many cases, numerical simulation methods of physical processes, based on the solution of differential equations in partial derivatives, are reasonable to implement.

Natural convection takes place in many technical systems. The solutions of various natural convection problems in nonconjugate [1-3] and conjugate [4-6] formulations were performed last years. The numerical analysis is conducted basically for the twodimensional problems of heat transfer, which is associated with the significant complexity when implementing the difference schemes for time-dependent three-dimensional conservation equations of mass, momentum, and energy. Investigations of threedimensional regimes of natural convection $[7,8]$ were mostly performed for nonconjugate problems. Moreover, the effect of radiation heat exchange on formation of differential and

* Corresponding author: nee alexander@mail.ru 
integral heat transfer characteristics was not analyzed in [7, 8]. Therefore, investigations of natural convection in a closed volume with radiant energy source in conjugate formulation are of great interest.

The main purpose of this study is numerical analysis of three-dimensional natural convection in a closed rectangular cavity under conditions of radiant heating and conjugate heat exchange.

\section{Problem formulation and solution method}

A typical infrared chamber were considered and presented in Fig. 1. Solution domain consisted of the air-filled cavity bounded by heat-conducting finite thickness walls. The radiant energy source was fixed on the top horizontal boundary. I assumed that the infrared emitter had time - independent surface temperature. Heat exchange with the environment was negligibly small at the external boundaries. The equalities of temperatures and heat fluxes were set at the solid - fluid interfaces. I assumed that the radiant energy coming from the infrared emitter was uniformly distributed along the bottom horizontal solid - fluid interface. Thermophysical properties of the system elements under study were considered as temperature independent. The flow regime was laminar.

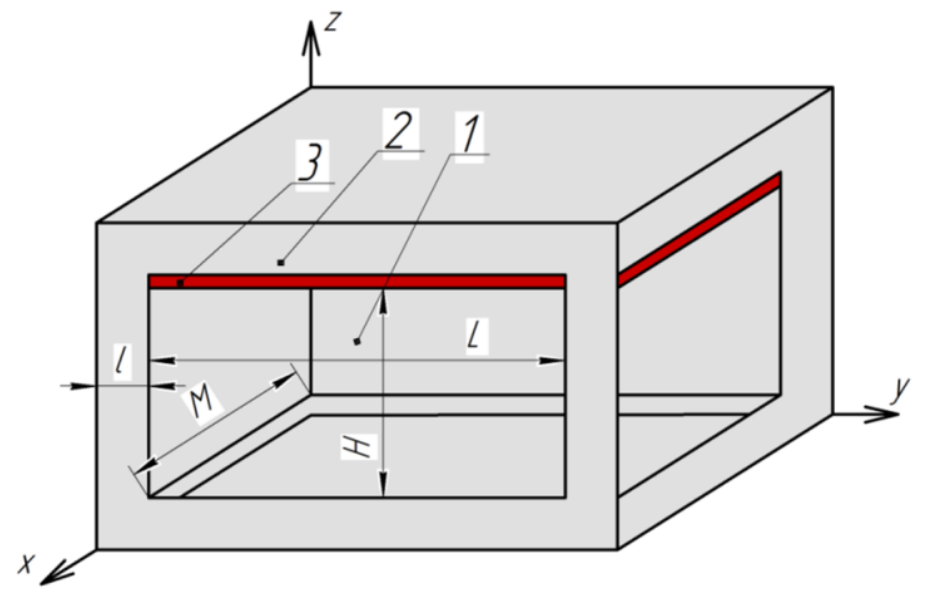

Fig. 1. Solution domain: 1 - air; 2 - walls; infrared emitter.

Heat transfer process under formulated physical model is described by unsteady threedimensional conservation equations mass, momentum, and energy. In terms of vorticity vector - vector potential - temperature dimensionless variables for viscous heat-conducting fluid, satisfying the Boussinesq approximation, these equations are as follow $[6,9]$ :

$$
\begin{gathered}
\frac{\partial \Omega_{x}}{\partial \tau}+U \frac{\partial \Omega_{x}}{\partial X}+V \frac{\partial \Omega_{x}}{\partial Y}+W \frac{\partial \Omega_{x}}{\partial Z}-\Omega_{x} \frac{\partial U}{\partial X}-\Omega_{y} \frac{\partial U}{\partial Y}-\Omega_{z} \frac{\partial U}{\partial Z}=\sqrt{\frac{\mathrm{Pr}}{\mathrm{Ra}}} \cdot \Delta \Omega_{x}+\frac{\partial \Theta_{1}}{\partial Y} \\
\frac{\partial \Omega_{y}}{\partial \tau}+U \frac{\partial \Omega_{y}}{\partial X}+V \frac{\partial \Omega_{y}}{\partial Y}+W \frac{\partial \Omega_{y}}{\partial Z}-\Omega_{x} \frac{\partial V}{\partial X}-\Omega_{y} \frac{\partial V}{\partial Y}-\Omega_{z} \frac{\partial V}{\partial Z}=\sqrt{\frac{\mathrm{Pr}}{\mathrm{Ra}}} \cdot \Delta \Omega_{y}-\frac{\partial \Theta_{1}}{\partial X} \\
\frac{\partial \Omega_{z}}{\partial \tau}+U \frac{\partial \Omega_{z}}{\partial X}+V \frac{\partial \Omega_{z}}{\partial Y}+W \frac{\partial \Omega_{z}}{\partial Z}-\Omega_{x} \frac{\partial W}{\partial X}-\Omega_{y} \frac{\partial W}{\partial Y}-\Omega_{z} \frac{\partial W}{\partial Z}=\sqrt{\frac{\operatorname{Pr}}{\operatorname{Ra}}} \cdot \Delta \Omega_{z} \\
\Delta \Psi_{x}=-\Omega_{x}, \Delta \Psi_{y}=-\Omega_{y}, \Delta \Psi_{z}=-\Omega_{z},
\end{gathered}
$$




$$
\begin{gathered}
\frac{\partial \Theta_{1}}{\partial \tau}+U \frac{\partial \Theta_{1}}{\partial X}-V \frac{\partial \Theta_{1}}{\partial Y}-W \frac{\partial \Theta_{1}}{\partial Z}=\frac{1}{\sqrt{\mathrm{Ra} \cdot \mathrm{Pr}}} \cdot \Delta \Theta_{1}, \\
\frac{1}{F O_{2}} \cdot \frac{\partial \Theta_{2}}{\partial \tau}=\Delta \Theta_{2}, \frac{1}{F O_{3}} \cdot \frac{\partial \Theta_{3}}{\partial \tau}=\Delta \Theta_{3} .
\end{gathered}
$$

The initial and boundary conditions for the equations (1)-(6) were set analogously to [4].

The boundary value problem (1)-(6) was solved by means of the finite-difference method $[10,11]$ on a uniform grid. $(71 \times 71 \times 71)$. Developed numerical code was tested on the benchmark problems of three-dimensional natural convection $[7,8]$. Comparison of the obtained results (isotherms and streamlines) showed their good agreement.

\section{Results and discussion}

Numerical analysis of three-dimensional conduction and natural convection was carried out for the following dimensionless criteria corresponding to laminar flow regime: Rayleigh number $R a=10^{6}$, Prandtl number $P r=0.71$, Kirpichev number $K i=13$, Fourier numbers $\mathrm{FO}_{2}=1.96 \cdot 10^{-8}, \mathrm{Fo}_{3}=9.5 \cdot 10^{-7}$. The results of conjugate heat exchange integral analysis are presented in terms of the mean Nusselt numbers at the bottom horizontal $\left(N u_{1}=\int_{0.1}^{1.1} \int_{0.1}^{1.1}\left|\frac{\partial \Theta}{\partial Z}\right|_{Z=0.1} d X d Y\right)$ and vertical $\left(N u_{2}=\int_{0.1}^{1.1} \int_{0.1}^{1.1}\left|\frac{\partial \Theta}{\partial Y}\right|_{Y=0.1} d X d Z\right)$ solid-fluid interfaces versus dimensionless time dependences and shown in Fig. 2.

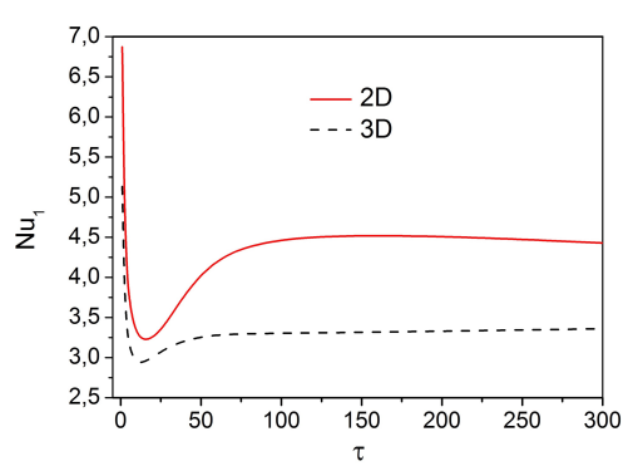

a

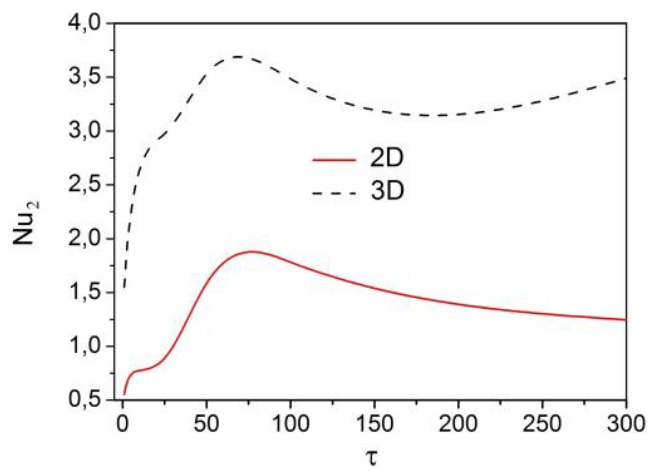

b

Fig. 2. Dependences of the mean Nusselt numbers versus dimensionless time at the: a) bottom horizontal solid-fluid interface; b) vertical solid-fluid interface.

It is clearly seen that the mean Nusselt numbers at the horizontal and vertical solid-fluid interfaces for both two and three dimensional problem formulations had similar trends. During the initial time period, $N u_{1}$ was reduced before $\tau$ was equal to 12 , which was associated with the formation of heated air layer at this interface. An increase in the mean Nusselt number at the bottom horizontal solid - fluid interface in a time range of 12 to 50 was due to the formation of circulating flows in the air cavity. Hereafter, $N u_{1}$ changed slightly, which was associated with the constant value of temperature gradient near the bottom horizontal wall. Along with that, an increase in dimensionless time led to a rise in the mean Nusselt number at the vertical solid - fluid interface, which was due to the 
increasing of air velocity along this interface. Obviously, this factor led to the intensification of heat exchange process. As the result, the mean Nusselt number increased. Higher values of $\mathrm{Nu}_{2}$ for the case of three-dimensional problem formulation was due to the more intensive heat loss from the heated air to the surfaces where vertical walls were connected. As a result, air velocity along the vertical solid-fluid interfaces was increased. This factor led to the $\mathrm{Nu}_{2}$ growth.

\section{Conclusion}

The mathematical model of three-dimensional heat transfer in a closed volume taking into account the basic relevant factors (conduction, convection, radiation and conjugate heat exchange) are suggested. Obtained results showed the possibility of further use of the approach $[6,9]$ when solving more complex problems of conjugate convective - radiative heat transfer in closed areas under conditions of the radiant heating.

The reported research was supported by Russian Federation President Grant for state support of the Russian Federation leading scientific schools SS-7538.2016.

\section{References}

1. D. Han, B. Yu., J. Chen, Y. Wang and Y. Wang, Int. Commun. Heat Mass, 68 (2015)

2. K. Szewc, J. Pozorski and A. Tanire, Int. J. Heat Mass Transf., 54 (2011)

3. Y. Wei, H.-S. Dou, Z. Wang, Y. Qian and W. Yan, Comput. Fluids, 124 (2016)

4. G. V. Kuznetsov and M. A. Sheremet, Int. J. Heat Mass Transf., 52 (2009)

5. E. Bilgen, Appl. Therm. Eng., 29 (2009)

6. G. V. Kuznetsov and M. A. Sheremet, Int. J. Heat Mass Transf., 54 (2011)

7. D. C. Lo, Int. Commun. Heat Mass, 37 (2010)

8. Z. Li, M. Yang, and Y. Zhang, Int. J. Heat Mass Transf., 94 (2016)

9. G. V. Kuznetsov and M. A. Sheremet, Int. J. Therm. Sci., 50 (2011)

10. G. V. Kuznetsov and A. E Nee, Thermophys. Aeromech., 23 (2016)

11. A. Nee IOP Conf. Ser. Mater. Sci. Eng., 93 (2015) 